\title{
Perfeccionismo y autoestima en estudiantes universitarios argentinos
}

\author{
Perfectionism and Self-esteem in Argentine \\ University Students
}

\author{
Gisela Paola Helguera ${ }^{1}$, Laura Beatriz $\operatorname{Oros}^{2} \bowtie$ \\ ${ }^{1}$ Universidad Adventista del Plata, Argentina \\ 2 Universidad de la Cuenca del Plata-Conicet; Universidad Adventista del Plata-isam
}

$\square$ Avenida Rusia 410, (335) Leandro N. Alem-Misiones. Correo electrónico: lauraorosb@gmail.com

Recibido: 2 de mayo del 2017 Aprobado: 3 de febrero del $2018 \quad$ Disponible en línea: 1 de abril de 2018

Cómo citar este artículo: Helguera, G. P., y Oros, L. B. (2018). Perfeccionismo y autoestima en estudiantes universitarios argentinos. Pensando Psicología, 14(23). doi: https://doi.org/10.16925/pe.v14i23.2266

\section{Resumen}

Objetivo: explorar en qué medida se relacionan el perfeccionismo y la autoestima en estudiantes universitarios argentinos.

Metodología: trescientos cuarenta y un sujetos respondieron la Almost Perfect Scale-Revised y la Escala de Autoestima de Rosenberg.

Resultados: Ios resultados mostraron una relación significativa, robusta y negativa entre la autoestima y la discrepancia perfeccionista $(r=-0,53 ; p<0,001)$, y débil pero significativa entre la autoestima y los altos estándares $(r=0,19 ; p<0,001)$. Asimismo, se observaron diferencias significativas $(F(2,145)=11,89 ; p<0,001 ; \eta 2 p=0,14)$ en la autoestima de los perfeccionistas adaptativos, en comparación con la de los perfeccionistas desadaptativos y los no perfeccionistas; los perfeccionistas adaptativos alcanzaron puntuaciones más altas que los otros dos grupos.

Conclusiones: se permitió establecer que, en consonancia con lo encontrado en estudios anteriores, las dimensiones del perfeccionismo se relacionan diferencialmente con la autoestima, de manera que es la discrepancia la característica que induce la mayor cantidad de variancia explicada. La importancia práctica de los resultados obtenidos justifica la incorporación de estrategias psicoeducativas para reducir el perfeccionismo disfuncional y fomentar el adaptativo en cualquier programa que tenga como objetivo la promoción de la autoestima en estudiantes universitarios argentinos.

Palabras clave: APS-R, autoestima, estudiantes universitarios argentinos, perfeccionismo. 


\title{
Perfectionism and Self-esteem in Argentine University Students
}

\begin{abstract}
Objective: To explore the extent to which perfectionism and self-esteem are related to Argentine university students.

Method: Three hundred and forty-one subjects answered the Almost Perfect Scale-Revised and the Rosenberg Self-Esteem Scale.

Results: The results showed a significant, robust, negative relationship between self-esteem and perfectionist discrepancy $(r=-0,53 ; p<0,001)$, and a weak but significant relationship between selfesteem and high standards $(r=0,19 ; p<0,001)$. Also, significant differences were observed $(F(2,145)=11,89 ; p<0,001, \eta 2 p=0.14)$ in the self-esteem of adaptive perfectionists, compared to that of maladaptive perfectionists and non-perfectionists; adaptive perfectionists scored higher than the other two groups.

Conclusions: It was established that, in line with the findings of previous studies, the dimensions of perfectionism are differentially related to self-esteem so that discrepancy is the characteristic that induces the greatest amount of variance explained. The practical importance of the results obtained justifies the incorporation of psychoeducational strategies to reduce dysfunctional perfectionism and foster adaptive perfectionism in any program whose aim is to promote self-esteem in Argentine university students.
\end{abstract}

Keywords: APS-R, self-esteem, Argentine university students, perfectionism.

\section{Perfeccionismo e autoestima em estudantes universitários argentinos}

\begin{abstract}
Resumo
Objetivo: explorar em que medida o perfeccionismo e a autoestima se relacionam em estudantes universitários argentinos.

Metodologia: 341 participantes responderam à Almost Perfect Scale-Revised e à Escala de Autoestima de Rosenberg.

Resultados: os resultados mostraram uma relação significativa, robusta e negativa entre a autoestima e a discrepância perfeccionista $(r=-0,53 ; p<0,001)$, e fraca, mas significativa, entre a autoestima e os altos padrões $(r=0,19 ; p<0,001)$. Do mesmo modo, diferenças significativas $(F(2,145)=$ $11,89 ; p<0,001 ; \eta 2 p=0,14)$ foram observadas na autoestima dos perfeccionistas adaptativos em comparação com a autoestima dos perfeccionistas desadaptativos e dos não perfeccionistas; os perfeccionistas adaptativos alcançaram pontuações mais altas do que os outros dois grupos.

Conclusões: foi possível estabelecer, em consonância com os resultados de estudos anteriores, que as dimensões do perfeccionismo se relacionam diferencialmente com a autoestima, de forma que a discrepância é a característica que induz a maior quantidade de variância explicada. A importância prática dos resultados obtidos justifica a incorporação de estratégias psicoeducacionais para reduzir 0 perfeccionismo disfuncional e fomentar 0 adaptativo em qualquer programa que tiver a promoção da autoestima em estudantes universitários argentinos como objetivo.
\end{abstract}

Palavras-chave: APS-R, autoestima, estudantes universitários argentinos, perfeccionismo. 


\section{Introducción}

Si bien el perfeccionismo ha sido tradicionalmente definido con base en sus características inflexibles, irracionales y patológicas, existen evidencias para reconocerlo como un constructo multidimensional que incorpora tanto aspectos adaptativos como disfuncionales (Frost, Heimberg, Holt, Mattia y Neubauer, 1993; Stoeber y Otto, 2006; Terry-Short, Owens, Slade y Dewey, 1995).

De acuerdo con LoCicero y Ashby (2000), es posible establecer una distinción entre los individuos no perfeccionistas, los perfeccionistas adaptativos y los disfuncionales, con base en dos dimensiones evaluables: los estándares de rendimiento y la discrepancia percibida entre estos estándares y el desempeño real. Los perfeccionistas desadaptativos han sido conceptualizados como personas con estándares muy elevados y tendencia a la autocrítica en sus autoevaluaciones, lo cual los lleva a percibir una alta discrepancia entre su desempeño real y aquel al cual pretendían llegar. Los perfeccionistas adaptativos, por el contrario, tienden a tener bajos niveles de autocrítica (baja discrepancia) aunque sus estándares de rendimiento son similares a los de los perfeccionistas desadaptativos. Finalmente, los individuos no perfeccionistas presentan estándares de rendimiento más bajos con relación a los dos grupos anteriores.

El perfeccionismo adaptativo se ha visto asociado a mayores niveles de felicidad, satisfacción, calidad de vida, bienestar psicológico y autoestima (Arana et al., 2010; Chan, 2012; Gnilka, Ashby y Noble, 2013; Grzegorek, Slaney, Franze y Rice, 2004; Öngen, 2009; Park y Jeong, 2015; Suh, Gnilka y Rice, 2017). En contraposición, estudios internacionales y de orden local han observado que el perfeccionismo desadaptativo se relaciona con diferentes variables perniciosas tales como: ansiedad (Ortega, Wang, Slaney, Hayes y Morales, 2014; Rice y Slaney, 2002), depresión (Elion, Wang, Slaney y French, 2012; Gnilka et al., 2013; Ortega et al., 2014; Wong, Chan y Lau, 2010-2011), desesperanza (Gnilka et al., 2013), suicidio (Kiamanesh, Dieserud y Haavind, 2015), desórdenes alimenticios (Ashby, Kottman y Schoen, 1998), baja calidad de vida percibida (Arana et al., 2010), baja autoestima (Elion, et al., 2012; Ortega et al., 2014; Rice, Ashby y Slaney, 1998; Rice y Dellwo, 2002; Rice y Slaney, 2002; Slaney, Rice, Mobley, Trippi y Ashby, 2001) y esquemas desadaptativos tempranos (Caputto, Cordero, Keegan y Arana; 2015), lo que provoca un impacto negativo en la salud tanto mental como física de las personas (Molnar, Reker, Culp, Sadava y DeCourville, 2006; Harrison y Craddock, 2016). De ahí que su prevalencia se asocie más bien a muestras de tipo clínicas que a poblaciones que no lo son (Egan, Wade y Shafran, 2011; Scotti, Sansalone, y Borda, 2011).

Una de las características psicológicas que estaría significativamente vulnerada en los perfeccionistas desadaptativos es la autoestima. Rosenberg (1965) la define como una actitud positiva o negativa hacia sí mismo que se delimita en función de criterios que varían para cada persona.

El buen desarrollo de la autoestima resulta fundamental, debido a que el modo en que un individuo se siente con respecto a sí mismo afecta de forma decisiva los demás aspectos de su experiencia e impacta significativamente en su salud mental (Anto y Jayan, 2016; Branden, 1995).

De forma similar a lo que sucede con el perfeccionismo, el nivel de autoestima de los individuos está ligado a un proceso de comparación en el cual intervienen valores y discrepancias. Una distancia mínima percibida entre el sí mismo ideal y el sí mismo real, da como consecuencia una autoestima saludable. En contraste, cuanto mayor es la distancia entre ambos elementos, menor es la autoestima, aun si la persona es vista positivamente por otros (Rosenberg, 1965). En este proceso de comparación cobra una importancia radical la autocrítica, la cual en los individuos con baja autoestima suele ser excesiva y conducir a la insatisfacción permanente (Baumeister, 1993).

Existen indicios de cómo ya en la infancia el déficit de autoestima puede estar acompañado de elevados niveles de perfeccionismo, autoexigencia y autocrítica (Junco-Herrera, 2010; Trujillo, 2010), lo cual puede deberse a que los individuos que presentan un perfeccionismo disfuncional tienden a asociar el alcance de metas con su sentido de sí mismos (DiBartolo, Frost, Chang, LaSota y Grills, 2004; Frost et al., 1993), presentan una baja aceptación incondicional de su persona (Flett, Besser, Davis y Hewitt, 2003) y desarrollan rumiaciones a modo de pensamientos automáticos y persistentes acerca de lo que deberían llegar a ser, que no hacen más que incrementar la distancia entre el sí mismo ideal y el sí mismo actual (Dixon, Earl, Lutz-Zois, Goodnight y Peatee, 2014).

De esta manera, el establecimiento de altos estándares propios del perfeccionismo (los cuales podrían 
equipararse con el sí mismo ideal de la persona) se relacionarían con la psicopatología solo cuando el alcance de estos fuera concebido como una condición necesaria para lograr y definir el sentido de estima personal del individuo (DiBartolo et al., 2004). Así, frente a experiencias de fracaso - que no ayudarían a afirmar la propia autoestima-, los perfeccionistas desadaptativos se verían expuestos a una gran aflicción psicológica (Flett et al., 2003).

Al conocer el papel de la discrepancia en la caracterización de ambos constructos, el impacto decisivo que pueden tener sobre la salud mental y la necesidad reconocida de contar con información local, actual y científicamente relevante que pueda dar lugar al desarrollo de planes e intervenciones para prevenir o promocionar la salud mental en población joven y adulta, resulta prometedor estudiar de qué manera se relacionan la autoestima y el perfeccionismo en estudiantes universitarios argentinos.

Como se mencionó, la revisión bibliográfica da cuenta de estudios previos que han analizado esta temática en jóvenes y adultos de otros contextos socioculturales (Elion et al., 2012; Ortega et al., 2014; Slaney et al., 2001), y concluyen que el perfeccionismo disfuncional se correlaciona negativa y significativamente con la autoestima. Según nuestro conocimiento, en el medio local el único trabajo que ha abordado esta problemática indica que sus resultados estarían en línea con los estudios anglosajones (Scotti et al., 2011). No obstante, por tratarse de un reporte breve no aporta datos estadísticos específicos ni menciona la magnitud o el tamaño del efecto de los hallazgos, lo cual resultaría crucial para determinar la importancia práctica de incluir abordajes psicoeducativos del perfeccionismo como medio para favorecer la autoestima en contextos universitarios.

Por tanto, el objetivo del presente estudio es complementar la investigación precedente a fin de conocer en qué medida se relacionan el perfeccionismo y la autoestima en jóvenes universitarios de nuestro país, con miras a determinar cuál sería la utilidad práctica de desarrollar programas de intervención para reducir el perfeccionismo disfuncional y promover el adaptativo como medio de potenciación de la autoestima en el medio universitario. Además, se propone establecer una diferenciación entre los perfeccionistas adaptativos, los desadaptativos y los no perfeccionistas, bajo la hipótesis de que la autoestima y el perfeccionismo no solo se relacionan robusta y significativamente, sino que además los perfeccionistas desadaptativos presentan menores puntajes de autoestima que los no perfeccionistas y los perfeccionistas adaptativos, y cómo la importancia práctica de estas diferencias es alta.

\section{Método}

\section{Participantes}

Se llevó a cabo un estudio de tipo descriptivo-correlacional en el que participaron voluntariamente 341 estudiantes universitarios (144 mujeres y 197 varones) pertenecientes a dos instituciones de gestión pública de la provincia de Santa Fe, Argentina. Las edades oscilaron entre los 17 y los 42 años de edad ( $M$ $=21,76 ; D E=2,81)$. La selección de los participantes se realizó mediante un muestreo de tipo no probabilístico intencional.

\section{Instrumentos}

\section{Almost Perfect Scale Revised APS-R (Slaney et al., 2001; adaptación, Arana, Keegan y Rutsz- tein, 2009)}

Esta escala, destinada a medir perfeccionismo, sigue un formato de respuesta tipo Likert; incluye 23 ítems distribuidos en tres subescalas: "Altos Estándares", "Orden" y "Discrepancia"; y posibilita distinguir perfiles desadaptativos y saludables de perfeccionismo.

La adaptación argentina realizada por Arana et al. (2009) con una muestra de estudiantes universitarios $(N=268)$ presenta coeficientes de discriminación aceptables, un nivel general de consistencia interna adecuado (alpha de Cronbach de 0,89) y una estructura factorial conservadora respecto de la escala original, de manera que emergen tres factores que explican el 53,35\% de la varianza. Los coeficientes alpha de Cronbach para cada dimensión fueron: 0,91 para discrepancia, 0,75 para altos estándares y 0,74 para orden. La primera dimensión mide el aspecto negativo del constructo y valora la magnitud en que los evaluados se autoperciben como incapaces de alcanzar sus propios estándares de desempeño, mientras que las dos últimas miden el aspecto positivo del perfeccionismo e indican la presencia de altos estándares de desempeño y la tendencia hacia el orden y la pulcritud. Los valores de alpha de Cronbach obtenidos por el presente estudio resultaron más bajos que los informados por Arana et al. (2009), pero aun así no desechables: $\alpha=0,86$ para "Discrepancia", $\alpha=0,69$ para "Altos estándares” y $\alpha=0,69$ para "Orden”. 
Escala de autoestima de Rosenberg (Rosenberg, 1965; adaptación, Góngora y Casullo, 2009)

Esta escala mide la autoestima siguiendo un modelo unidimensional. Consta de 10 ítems (cinco directos y cinco inversos) con cuatro alternativas de respuesta que oscilan entre "muy de acuerdo" y "muy en desacuerdo", lo cual permite diferenciar entre individuos con mayor o menor autoestima.

En la validación realizada en Argentina por Góngora y Casullo (2009) en una población general $(N=313)$ y otra clínica $(N=60)$, se observó que la escala se ajustaba bien al modelo unidimensional en los dos grupos evaluados. La consistencia interna resultó adecuada (alpha de Cronbach 0,70 y 0,78), y se evidenció una buena validez divergente con escalas de ansiedad y depresión, y convergente con subescalas de inteligencia emocional. El alpha de Cronbach obtenido por el presente estudio fue de $\alpha=0,80$.

\section{Procedimientos de recolección $\mathrm{y}$ análisis de datos}

Los estudiantes fueron contactados en las universidades, previa solicitud de autorización a los directivos de dichas instituciones educativas. La evaluación fue colectiva dentro del horario académico y se le brindó a cada estudiante una hoja de consentimiento informado que incluía toda la información pertinente con respecto a la investigación, además de las escalas para la medición del perfeccionismo y la autoestima anteriormente mencionadas. Asimismo, se les garantizó el completo anonimato y una manipulación ética y responsable de los datos que pudieran brindar.

Con respecto a los análisis, los datos se estudiaron con relación a su normalidad, homocedasticidad e independencia como paso previo para proceder a la ejecución de las pruebas estadísticas. Se efectuaron correlaciones bivariadas con el objetivo de conocer la relación entre ambas variables, además de un análisis de regresión lineal múltiple por pasos sucesivos en el que se incluyeron como predictoras las tres dimensiones de perfeccionismo ("Altos estándares", "Orden" y "Discrepancia"), y como variable de respuesta a los valores de autoestima. También se ejecutó un análisis de variancia simple (Anova), con comparaciones post hoc mediante el método de Scheffé, a fin de contrastar la autoestima entre los perfeccionistas adaptativos, los desadaptativos y los no perfeccionistas. Para esto, los estudiantes se clasificaron según los valores obtenidos en las dimensiones "Altos estándares" y "Discrepancia”. Así, se consideraron no perfeccionistas a aquellos que puntuaron bajo en "Altos estándares", se seleccionaron como perfeccionistas desadaptativos a aquellos que puntuaron alto en las dos dimensiones, y como perfeccionistas adaptativos a aquellos que puntuaron alto en "Altos estándares" y bajo en "Discrepancia". Se consideraron valores altos y bajos de estándares a aquellos que caían por encima y por debajo del percentil 67 , respectivamente, $y$ valores altos y bajos de discrepancia a aquellos que se situaban por encima y por debajo de la mediana, respectivamente, tal como se ha sugerido en estudios anteriores (Ashby y Kottman, 1996; LoCicero y Ashby, 2000). Si bien este es un método aceptado, existen otros criterios comparables de clasificación (i.e. análisis de clústeres), que podrían considerarse en próximos estudios (Rice y Slaney, 2002; Rice y Ashby, 2007). El tamaño del efecto se calculó mediante el estadístico $\eta^{2} p$ (Cohen, 1988), considerando los siguientes valores de referencia: (a) 0,01= tamaño pequeño; (b) 0,06 = tamaño mediano; y (c) 0,14 = tamaño grande (Cárdenas-Castro y ArancibiaMartini, 2014).

El análisis de datos perdidos indicó que seis ítems de perfeccionismo presentaron omisiones ligeramente superiores al 5\% (6\% en todos los casos). Los casos (sujetos) que cumplieron con esta condición fueron excluídos de la muestra para los posteriores análisis estadísticos. El procesamiento de los datos se efectuó con el programa estadístico spss, versión 18.0.

\section{Resultados}

El análisis de correlación bivariada puso de manifiesto que la autoestima está fuertemente relacionada con la dimensión "discrepancia del perfeccionismo" $(r=-0,53 ; p<0,001)$, y débilmente - aunque de forma significativa - con la de "altos estándares" $(r=0,19 ; p<0,001)$. La dimensión "orden" mostró una correlación baja positiva con la autoestima, cercana a la significación mínima requerida $(r=0,10$; $p=0,065$ ).

A partir de este análisis, se pretendió realizar un estudio de predicción para establecer qué porcentaje de la autoestima estaba explicada por el perfeccionismo y cuánta variancia inducía cada dimensión de este. A pesar de que el factor orden no mostró una correlación significativa se decidió incluirlo a modo exploratorio en el modelo de regresión debido a que 
el margen de error está cercano al aceptable, y a que existe muy poca información respecto al comportamiento de estas dos variables en el medio local y su inclusión podría resultar un aporte útil en materia de tendencias de análisis para futuros estudios.

Con relación a los supuestos del modelo de regresión, se constató que no existía relación lineal exacta entre las variables independientes. Los valores de tolerancia resultaron satisfactorios (superiores a 0,80 ) y los factores de inflación de la varianza (FIV) estuvieron en torno a 1 (siendo 10 el máximo admisible). Se observó solo un índice de condición superior a 15 (IC $=20,07)$, pero aun así dentro de los límites aceptables (Pardo y Ruiz, 2005). Con relación a la independencia, el estadístico de Durbin-Watson se situó dentro del intervalo $1,5-2,5$, lo cual indica que los residuos eran independientes $(D W=2,04)$. Finalmente, se analizó el gráfico de probabilidad normal, observándose que los puntos se alineaban sobre la diagonal, lo cual indica que los residuos tendían a distribuirse normalmente.

El análisis de regresión lineal por pasos indicó que el modelo de mejor predicción incluye las tres dimensiones del perfeccionismo, aunque el aporte de la dimensión "Discrepancia" supera ampliamente al de "Altos estándares" y "Orden" (véase la tabla 1).
Por otra parte, con respecto a la clasificación de los sujetos a partir de los valores de estándares personales y discrepancia, se encontraron: (a) 232 individuos no perfeccionistas, (b) 60 perfeccionistas desadaptativos, y (c) 48 adaptativos. Si bien estas proporciones se asemejan a las reportadas en un estudio anterior con universitarios argentinos (Arana et al., 2014), y reflejan probablemente la distribución natural de la población, a fin de evitar comparaciones sesgadas se determinó, a riesgo de perder potencia, reducir el número de los grupos (a) y (b) y así conseguir una mayor equivalencia de tamaño. La eliminación de los casos fue completamente aleatoria según los procedimientos que ofrece el programa estadístico spss. De esta manera, los grupos quedaron conformados como sigue: (a) no perfeccionistas $(n=50)$, (b) perfeccionistas desadaptativos $(n=50)$, y (c) perfeccionistas adaptativos $(n=48)$. Con el propósito de estudiar el supuesto de normalidad de la variable dependiente en estas submuestras se calculó el estadístico ShapiroWilk. Como puede observarse en la tabla 2, los tres grupos tienen asociados niveles críticos mayores al 0,05 , lo cual indica que los datos proceden de poblaciones normales. Asimismo, el test de Levene mostró que se cumplía con el criterio de homogeneidad de variancias $(F(2,145)=0,33 ; p=0,718)$, por lo cual se

Tabla 1

Dimensiones del perfeccionismo como predictores de la autoestima

\begin{tabular}{cccccc}
\hline Perfeccionismo & $\boldsymbol{R}^{\mathbf{2}}$ & $\boldsymbol{F}$ & $\Delta \boldsymbol{R}^{2}$ & $\boldsymbol{\beta}$ & IC 95\% \\
\hline Paso 1 Discrepancia & 0,28 & $131,34^{* * *}$ & 28 & $-0,53^{* * *}$ & {$[-0,20,-0,14]$} \\
\hline Paso 2 Discrepancia & 0,34 & $87,14^{* * *}$ & 0,06 & $-0,56^{* * *}$ & {$[-0,20,-0,15]$} \\
Altos estándares & & & & $0,25^{* * *}$ & {$[0,12,0,26]$} \\
\hline Paso 3 Discrepancia & 0,35 & $60,62^{* * *}$ & 0,01 & $-0,57^{* * *}$ & {$[-0,21,-0,15]$} \\
Altos estándares & & & & $0,21^{* * *}$ & {$[0,09,0,23]$} \\
Orden & & & & $0,11^{*}$ & {$[0,02,0,21]$} \\
\hline
\end{tabular}

Nota. Elaboración propia

Tabla 2

Test de Shapiro-Wilk para el estudio de normalidad de los datos

\begin{tabular}{llccc}
\hline & \multicolumn{2}{c}{ Clasificación de perfeccionismo } & \multicolumn{2}{c}{ Shapiro-Wilk } \\
\hline \multirow{3}{*}{ Autoestima } & & Estadístico & gl & Sig. \\
& NP & 0,969 & 50 & 0,203 \\
& PA & 0,974 & 48 & 0,363 \\
& PD & 0,981 & 50 & 0,611 \\
\hline
\end{tabular}

Nota. Elaboración propia. NP se corresponde con no perfeccionista, PA con perfeccionista adaptativo y PD con perfeccionista desadaptativo 
procedió a utilizar la prueba paramétrica Anova con comparaciones post hoc de Scheffé.

Los resultados de este análisis mostraron que la clasificación perfeccionista incide sobre la autoestima de los estudiantes con un tamaño de efecto alto $\left(F(2,145)=11,89 ; p<0,001 ; \eta^{2} p=0,14\right)$ y que las diferencias significativas se producen en las comparaciones de los perfeccionistas adaptativos con los desadaptativos $(p<0,001)$, y con los no perfeccionistas $(p=0,001)$. De acuerdo con el análisis de medias, los perfeccionistas adaptativos alcanzarían una mayor autoestima con relación a los demás estudiantes (véase la tabla 3).

\section{Discusión y conclusiones}

El propósito de este estudio fue evaluar en qué medida se relacionaban la autoestima y el perfeccionismo en estudiantes universitarios de Argentina. Los resultados indicaron que la autoestima de los estudiantes universitarios se ve afectada negativamente por el perfeccionismo desadaptativo, lo cual llevaría a ratificar la necesidad de incluir intervenciones que permitan disminuir esta disfunción cognitiva si se desean optimizar los resultados de cualquier programa de promoción de la estima personal. Asimismo, se observó que el perfeccionismo adaptativo funcionaría como un posible inductor de los sentimientos de valía personal.

Puntualmente, los resultados indicaron que los sujetos con una alta discrepancia son proclives a presentar una autoestima baja. Estos resultados están en línea con estudios realizados en otras latitudes, siendo la magnitud de la correlación observada en nuestro trabajo más alta $(r=-0,53)$ con respecto a la reportada por Slaney et al. (2001) en estudiantes norteamericanos con ascendencia predominantemente europea ( $r$ entre -0,35 y -0,44); y por Elion et al. (2012) en estudiantes norteamericanos con ascendencia africana $(r=-0,45)$, pero similar a la encontrada por Ortega et al. (2014) en estudiantes norteamericanos con ascendencia latina $(r=-0,55)$.
Los individuos caracterizados por este aspecto desadaptativo del perfeccionismo resultan más propensos a tener una baja aceptación incondicional de sí mismos y una baja autoestima (Flett et al., 2003; DiBartolo, Yen Li y Frost, 2007; Rice y Slaney, 2002), dado que tienden a asociar el alcance de las metas propuestas con su sentido de sí mismos (DiBartolo et al., 2004; Frost et al., 1993). De este modo, la presencia en estos sujetos de la percepción de una brecha entre el logro perseguido y su desempeño real constituye una experiencia negativa, que se interpreta como un indicador de la propia falta de valía que los expone a la angustia psicológica (Hewitt y Dyck, 1986; Frost et al., 1993; Flett et al., 2003).

De esta manera, una interpretación cognitiva errónea lleva a los individuos con alta discrepancia a considerar que ninguna acción realizada resulta lo suficientemente buena como para alcanzar sus desmesurados estándares de actuación personal, de forma que influye este sentimiento subjetivo de fracaso directamente sobre su propio sentido de valor personal o autoestima. Por otra parte, esta necesidad extrema experimentada por los perfeccionistas desadaptativos de llevar a la máxima expresión de excelencia posible sus elevados estándares de actuación y metas personales también podría ser interpretada como una búsqueda de sobrevaloración destinada a cubrir, mediante la admiración y la aprobación de los demás, los vacíos personales existentes en cuanto a sentimientos de amor y valoración propios de la persona (Cha, 2016).

Los resultados correlacionales también revelaron que los sujetos con un puntaje elevado en la subescala "Altos estándares" tienden a mostrar una autoestima alta, observándose la misma tendencia con respecto al factor "Orden", aunque en este último caso sin llegar a la significación estadística requerida. Los estudios de Slaney et al. (2001) y Eliot et al. (2012) reportaron correlaciones significativas entre "Autoestima" y "Altos estándares", de igual magnitud o muy cercanas ( $r=0,19 ; r=0,22$; respectivamente)

Tabla 3

Valores promedio de la variable autoestima según la clasificación perfeccionista

\begin{tabular}{ccccccc}
\hline Clasificación & $\boldsymbol{M}$ & $\boldsymbol{n}$ & $\boldsymbol{D E}$ & $\boldsymbol{F}$ & $\boldsymbol{p}$ & Post hoc $(\boldsymbol{p})$ \\
\hline No perfeccionista & $30,90^{\mathrm{a}}$ & 50 & 3,501 & 11,89 & 0,000 & a-b $(0,001)$ \\
Perf. adaptativo & $33,73^{\mathrm{b}}$ & 48 & 3,595 & & a-c $(0,855)$ \\
Perf. desadaptativo & $30,50^{\mathrm{c}}$ & 50 & 3,598 & & b-c $(0,000)$ \\
\hline
\end{tabular}

Nota. Elaboración propia 
a la informada en el presente estudio $(r=0,19)$. Con referencia a la subescala de "Orden", la baja magnitud de la relación observada aquí $(r=0,10)$, también se asemeja a las reportadas por Eliot et al. (2010) $(r=0,12)$ y Slaney et al. (2001) $(r=0,05$ y $r=0,14)$, de modo que resulta significativa solo para la última correlación dentro del paréntesis.

Estos hallazgos, entonces, se muestran en consonancia con la información obtenida en estudios anteriores con población anglosajona, en los que se ha visto que los altos estándares están asociados a una buena autoestima y a emociones y resultados mentales más positivos, e indican mejor salud psicológica en general (Ashby y Rice, 2002; DiBartolo et al., 2007; Rice y Stuart, 2010), por lo que las expectativas de rendimiento elevado pueden tomarse como un indicador de una búsqueda funcional o positiva de excelencia (Rice y Stuart, 2010). De este modo, esta cualidad podría ser utilizada como una herramienta adecuada para mejorar el desempeño, lograr el alcance de las metas y mejorar el sentido de valía personal. Asimismo, también sería correcto destacar que las personas que gozan de un alto grado de autoestima son las que presentan la confianza en sí mismas, el amor propio y la aceptación incondicional necesarios para el establecimiento de altos estándares de actuación personal; cuanto más alta es la autoestima, más ambicioso se tiende a ser (Branden, 1995), por lo cual estas variables podrían retroalimentarse positivamente.

Por otra parte, los resultados obtenidos en el análisis de variancia simple con comparaciones post hoc confirman que, si bien los perfeccionistas desadaptativos presentan menores puntajes de autoestima con relación a los no perfeccionistas y los perfeccionistas adaptativos, la diferencia entre los dos primeros es insignificante. Esto quiere decir que la autoestima se ve igualmente fragilizada tanto en el caso de aquellos sujetos que muestran un perfeccionismo pernicioso como en el de aquellos que no esperan mucho de sí mismos, se imponen estándares poco elevados y no están interesados en esforzarse o dar lo mejor de sí para alcanzar la excelencia. El tamaño del efecto para estos resultados fue alto, aunque algo menor que los reportados por Elion et al. $(2012)\left(\eta^{2} \mathrm{p}=0,16\right)$ y Ortega et al. (2013) $\left(\eta^{2} \mathrm{p}=0,19\right)$, lo cual podría deberse a que el tamaño de las submuestras del presente estudio fue menor que en los casos mencionados.

Específicamente con respecto a los perfeccionistas desadaptativos, los resultados se corresponden con las nociones anteriormente planteadas. Los perfeccionistas disfuncionales padecen de una aceptación condicional de sí mismos que los lleva a concebir su valor como personas de acuerdo con los logros alcanzados, razón por la cual procuran una hipercompetitividad en la que todo lo que se realice tiene que ser excelente (Ellis, 2002). La disfunción que estos presentan los lleva a desmerecer su actuación personal y es empleada del mismo modo en detrimento de su autoestima. Cuando el individuo transforma su deseo de alcanzar el éxito en demandas que deben ser cumplidas, tiende a considerarse indigno del amor y la aprobación de otros en caso de no cumplir con sus objetivos (Ellis, 2002). De este modo, los estándares elevados nunca llegan a percibirse como consumados dado que una autocrítica excesiva respecto al desempeño ejecutado lleva a los sujetos a colocarse en un lugar siempre inferior al que aspiraban para, posteriormente, provocar un aumento excesivo de dichos estándares cuyo cumplimiento otorgue sentimientos de satisfacción y bienestar, y quede de esta manera conformado un circuito autodesvalorizador (De Rosa, Valle, Rutsztein y Keegan, 2012).

La baja discrepancia en los perfeccionistas adaptativos es la razón por la cual sus niveles de autoestima son mejores. Sus actitudes frente a las eventuales fallas son adaptativas: el fracaso no les genera sentimientos de derrota y desolación, sino que provee una fuerte motivación para el crecimiento y el aprendizaje (Kottman y Ashby, 2000). Esta actitud flexible - que se contrapone marcadamente a la rigidez que caracteriza a los perfeccionistas irracionales - auguraría un mejor desempeño y una mayor estima personal. Al mismo tiempo, estos sujetos se manejan con estándares de desempeño razonablemente elevados, lo que los protegería de caer en la mediocridad, el estancamiento y el conformismo, elementos que también podrían considerarse perniciosos para el desarrollo de la valoración personal.

En concordancia con lo mostrado en investigaciones previas, es posible afirmar entonces que los estándares elevados resultan beneficiosos a menos que estén unidos con una tendencia permanente a la autocrítica, o a percibir una brecha dolorosa entre las expectativas y la evaluación del desempeño propio (Rice y Stuart, 2010). En tanto los altos estándares no se presenten a modo de expectativas irreales y no se acompañen de severas críticas ante el fracaso, estos pueden no solo no generar inconvenientes, sino además llegar a considerarse una fuente de motivación para la acción (Kottman y Ashby, 2000).

De esta manera, se concluye que mientras la discrepancia tiende a asociarse a una baja autoestima y, por consiguiente, a aumentar el posible riesgo 
psicopatológico, los altos estándares se vinculan con una autoestima elevada que daría como resultado un mejor funcionamiento psicológico.

Lo planteado hasta el momento sugiere que el desarrollo de un perfeccionismo adaptativo y de una buena autoestima van de la mano y resultan en un gran beneficio para la salud psicológica de los estudiantes. De ahí que no solo resulte oportuno disminuir o prevenir el perfeccionismo disfuncional, sino también promover el adaptativo, a fin de lograr un mejor ajuste psicológico. Estas consideraciones deberían tenerse en cuenta especialmente al momento de elaborar planes y proyectos tendientes a mejorar las creencias y emociones referidas a la propia persona. Al respecto puede consultarse el trabajo de Arana, Miracco, Galarregui y Keegan (2017), quienes han implementado y evaluado la eficacia de una intervención breve para la reducción de las preocupaciones perfeccionistas y el distrés psicológico en estudiantes universitarios.

En cuanto a las limitaciones del presente estudio cabe destacar las particulares características de la muestra, en cuanto a edad y nivel de instrucción, y el sistema de muestreo elegido, debido a lo cual la generalización de las conclusiones extraídas debería restringirse a contextos universitarios locales. Además, debe tenerse en cuenta que el tamaño de cada grupo de contrastación (perfeccionistas adaptativos, perfeccionistas desadaptativos y no perfeccionistas) fue relativamente pequeño, por lo cual sería deseable en próximos estudios recoger una mayor cantidad de participantes. De todas formas, vale la pena mencionar cómo a pesar de esta limitación - que podría poner en riesgo la potencia estadística- el tamaño de los efectos observados ha sido alto, lo cual indica que, efectivamente - como se había hipotetizado-, la relación entre las variables es lo suficientemente robusta y digna de atención por parte de los equipos de intervención psicológica en las entidades educativas. Finalmente, sería interesante en futuras investigaciones analizar la pertinencia de incluir en la clasificación perfeccionista un cuarto subtipo (identificado por bajos estándares y alta discrepancia), más recientemente propuesto por Gaudreau y Thompson (2010), ya que si bien su inclusión no está libre de debates, existen antecedentes que indican cómo podría aportar información relevante a la comprensión de este complejo constructo y su relación con otras variables de la salud mental (Gaudreau, 2012; Wang, Slaney y Rice, 2007).

\section{Referencias}

Anto, S. P. y Jayan, C. (2016). Self-esteem and Emotion Regulation as Determinant of Mental Health of Youth. SIs Journal of Projective Psychology \& Mental Health, 23(1), 34-40. Recuperado de https://goo.gl/GicziZ

Arana, F. G., Galarregui, M. S., Miracco, M. C., Partarrieu, A. I., De Rosa, L., Lago, A. (...) y Keegan, E. G. (2014). Perfeccionismo y desempeño académico en estudiantes universitarios de la Ciudad Autónoma de Buenos Aires. Acta Colombiana de Psicología, 17(1), 71-77. Recuperado de https://goo.gl/LG15n7

Arana, F. G., Keegan, E. G. y Rutsztein, G. (2009). Adaptación de una medida multidimensional de perfeccionismo: la Almost Perfect Scale-Revised (APS-R). Un estudio preliminar sobre sus propiedades psicométricas en una muestra de estudiantes universitarios argentinos. Evaluar, 9, 35-53. Recuperado de https:// goo.gl/bGEHFy

Arana, F. G., Miracco, M. C., Galarregui, M. S. y Keegan, E. G. (2017). A Brief Cognitive Behavioural Intervention for Maladaptive Perfectionism in Students: A Pilot Study. Behavioural and Cognitive Psychotherapy, 45(5), 537-542. Recuperado de https://goo.gl/uyqAGv

Arana, F. G., Scappatura, M. L., Miracco, M., Elizathe, L., Rutsztein, G., y Keegan, E. (2010). Perfeccionismo positivo/negativo y calidad de vida percibida en estudiantes de psicología, medicina e ingeniería de la ciudad de Buenos Aires. Anuario de investigaciones, 17, 19-24. Recuperado de https://goo.gl/ede8FP

Ashby, J. y Kottman, T. (1996). Inferiority as a Distinction Between Normal and Neurotic Perfectionism. Individual Psychology, 52, 237-245. Recuperado de https:// goo.gl/EdgT2C

Ashby, J., Kottman, T. y Schoen E. (1998). Multidimensional Perfectionism and Eating Disorders. Journal of Mental Health Counseling, 20, 261-271.

Ashby, J. S. y Rice, K. G. (2002). Perfectionism, Dysfunctional Attitudes, and Self-esteem: A Structural Equations Analysis. Journal of Counseling and Development, 80(2), 197-203. doi: http://dx.doi.org/10.1002/ j.1556-6678.2002.tb00183.x

Baumeister, R. F. (1993). SSelf-esteem: The Puzzle of Low Self-Regard. Nueva York: Plenun.

Branden, N. (1995). Cómo mejorar su autoestima. México: Paidós.

Caputto, I., Cordero, S., Keegan, E. y Arana, F. (2015). Perfeccionismo y esquemas desadaptativos tempranos: un 
estudio con estudiantes universitarios. Ciencias Psicológicas, 9(2), 245-257. Recuperado de https://goo.gl/ Jqtq2R

Cárdenas-Castro, M. y Arancibia-Martini, H. (2014). Potencia estadística y cálculo del tamaño de efecto en ${ }^{*}$ POWER: complementos a las pruebas de significación estadística y su aplicación en Psicología. Salud \& Sociedad, 5(2), 210-224. Recuperado de https://goo. $\mathrm{gl} / \mathrm{j} 6 \mathrm{pAZH}$

Cha, M. (2016). The Mediation Effect of Mattering and Self-esteem Between Socially Prescribed Perfectionism and Depression Based on the Social Disconnection Model. Personality and Individual Differences, 88, 148159. doi: http://dx.doi.org/10.1016/j.paid.2015.09.008

Chan, D. W. (2012). Life Satisfaction, Happiness and the Growth Mindset of Healthy and Unhealthy Perfectionists Among Hong Kong Chinese Gifted Students. Roeper Review, 34(4), 224-233. Recuperado de https:// goo.gl/PuKddg

Cohen, J. (1988). Statistical Power Analysis for The Behavioral Sciences. Hillsdale, NJ: Lawrence Earlbaum Associates.

De Rosa, L., Valle, A. D., Rutsztein, G. y Keegan, E. (2012). Perfeccionismo y autocrítica: consideraciones clínicas. Revista Argentina de Clínica Psicológica, 21(3), 209 215. Recuperado de https://goo.gl/ffWEoP

DiBartolo, P. M., Frost, R. O., Chang, P., LaSota, M. y Grills, A. E. (2004). Shedding Light on the Relationship Between Personal Standards and Psychopathology: The Case of Contingent Self-worth. Journal of Rational-Emotive and Cognitive Behavior Therapy, 22, 241254. doi: http://dx.doi.org/10.1023/B:JORE.00000473 10.94044.ac

DiBartolo, P. M., Yen Li, C. y Frost, R. O. (2007). How Do the Dimensions of Perfectionism Relate to Mental Health? Cognitive Therapy and Research, 32, 401-417. doi: http://dx.doi.org/10.1007/s10608-007-9157-7

Dixon, L. J., Earl, K. A., Lutz-Zois, C. J., Goodnight, J. A. y Peatee, J. J. (2014). Explaining the Link Between Perfectionism and Self-Forgiveness: The Mediating Roles of Unconditional Self-acceptance and Rumination. Individual Differences Research, 12(3), 101-111. Recuperado de https://goo.gl/oQDNXr

Egan, S. J., Wade, T. D. y Shafran, R. (2011). Perfectionism as a Transdiagnostic Process: A Clinical Review. Clinical Psychology Review, 31(2), 203-212. doi: http://dx.doi. org/10.1016/j.cpr.2010.04.009

Elion, A. A., Wang, K. T., Slaney, R. B. y French, B. H. (2012). Perfectionism in African American Students: Relationship to Racial Identity, GPA, Self-esteem, and
Depression. Cultural Diversity and Ethnic Minority Psychology, 2, 118-127. Recuperado de http://dx.doi. org/10.1037/a0026491

Ellis, A. (2002). The Role of Irrational Beliefs in Perfectionism. En G. L. Flett, y P. L. Hewitt (Eds.), Perfectionism: Theory, Research and Treatment (pp. 181-198). Washington DC: American Psychological Association.

Flett, G. L., Besser, A., Davis, R. A. y Hewitt, P. L. (2003). Dimensions of Perfectionism, Unconditional Self-acceptance, and Depression. Journal of Rational-Emotive and Cognitive Behavior Therapy, 21, 119-138. Recuperado de https://goo.gl/Biji99

Frost, R. O., Heimberg, R. G., Holt, C. S., Mattia, J. L. y Neubauer, A. L. (1993). A Comparison of Two Measures of Perfectionism. Personality and Individual Differences, 14, 119-126. doi: http://dx.doi.org/10.1016/01918869(93)90181-2

Gaudreau, P. (2012). A Methodological Note on The Interactive and Main Effects of Dualistic Personality Dimensions: An Example Using the 2 x 2 Model of Perfectionism. Personality and Individual Differences, 52(1), 26-31. doi: http://dx.doi.org/10.1016/j. paid.2011.08.022

Gaudreau, P. y Thompson, A. (2010). Testing a 2 x 2 Model of Dispositional Perfectionism. Personality and Individual Differences, 48(5), 532-537. doi: http://dx.doi. org/10.1016/j.paid.2009.11.031

Gnilka, P. B., Ashby, J. S. y Noble, C. M. (2013). Adaptive and Maladaptive Perfectionism as Mediators of Adult Attachment Styles and Depression, Hopelessness, and Life Satisfaction. Journal of Counseling \& Development, 91, 78-86. doi: http://dx.doi.org/10.1002/j.15566676.2013.00074.x

Góngora, V. C. y Casullo, M. M. (2009). Validación de la escala de autoestima de Rosenberg en población general y en población clínica de la ciudad de Buenos Aires. Ridep, 27(1), 179-194. Recuperado de https:// goo.gl/fi73Yd

Grzegorek, J. L., Slaney, R. B., Franze, S. y Rice, K. G. (2004). Self-criticism, Dependency, Self-Esteem and Grade Point Average Satisfaction Among Clusters of Perfectionists and Nonperfectionists. Journal of Counseling Psychology, 51(2), 192-200. doi: http://dx.doi. org/10.1037/0022-0167.51.2.192

Harrison, F. y Craddock, A. E. (2016). How Attempts to Meet Other/s Unrealistic Expectations Affect Health: Health-Promoting Behaviours as a Mediator Between Perfectionism and Mental Health. Psychology, Health 
and Medicine, 21(3), 386-400. Recuperado de https:// goo.gl/c73bes

Hewitt, P. L. y Dyck, D. G. (1986). Perfectionism, Stress and Vulnerability to Depression. Cognitive Therapy and Research, 10, 137-142. doi: http://dx.doi.org/10.1007/ BF01173389

Junco-Herrera, I. (2010). Autoestima: me gusta como soy, confío en mí. Temas para la Educación: Revista Digital para Profesionales de la Enseñanza, 9, 1-15. Recuperado de https://goo.gl/6E1uyZ

Kiamanesh, P., Dieserud, G. y Haavind, H. (2015). From a Cracking Façade to a Total Escape: Maladaptive Perfectionism and Suicide. Death Studies, 39, 316-322. Recuperado de https://goo.gl/ZA14Xj

Kottman, T. y Ashby, J. (2000). Perfectionistic Children and Adolescents: Implications for School Counselors. Professional School Counseling, 3(3), 182-188. Recuperado de https://goo.gl/zz8g3z

LoCicero, K. A. y Ashby, J. S. (2000). Multidimensional Perfectionism and Self-Reported Self-efficacy in College Students. Journal of College Student Psychotherapy, 15(2), 47-56. doi: http://dx.doi.org/10.1300/ J035v15n02_06

Molnar, D. S., Reker, D. L., Culp, N. A., Sadava, S. W. y DeCourville, N. H. (2006). A Mediated Model of Perfectionism, Affect, and Physical Health. Journal of Research in Personality, 40(5), 482-500. doi: http://dx. doi.org/10.1016/ j.jrp.2005.04.002

Öngen, D. E. (2009). The Relationship Between Perfectionism and Multidimensional Life Satisfaction Among High School Adolescents in Turkey. Journal of Multicultural Counseling and Development, 37(1), 52-64. Recuperado de https://goo.gl/9p8N8t

Ortega, N. E, Wang, K. T., Slaney, R. B. Hayes, J. A. y Morales, A. (2014). Personal and Familial Aspects of Perfectionism in Latino/a Students. The Counseling Psychologist, 42(3), 406-427. doi: http://dx.doi.org/10.1177/ 0011000012473166

Pardo, A. y Ruiz, M. A. (2005). Análisis de datos con SPSS 13 base. Madrid: McGraw-Hill.

Park, H. y Jeong, D. Y. (2015). Psychological Well-being, Life Satisfaction, and Self-esteem Among Adaptive Perfectionists, Maladaptive Perfectionists, and Nonperfectionists. Personality and Individual Differences, 72, 165-170. doi: http://dx.doi.org/10.1016/j. paid.2014.08.031

Rice, K. G. y Ashby, J. (2007). An Efficient Method for Classifying Perfectionists. Journal of Counseling
Psychology, 54(1), 72-85. Recuperado de http://dx.doi. org/10.1037/0022-0167.54.1.72

Rice, K. G., Ashby, J. S. y Slaney, R. B. (1998). Self-Esteem as a Mediator Between Perfectionism and Depression: A Structural Equations Analysis. Journal of Counseling Psychology, 45, 304-314. doi: http://dx.doi. org/10.1037/0022-0167.45.3.304

Rice, K. G. y Dellwo, J. P. (2002). Perfectionism and Self-Development: Implications for College Adjustment. Journal of Counseling \& Development, 80, 188-196. doi: http://dx.doi.org/10.1002/j.1556-6678.2002.tb00182.x

Rice, K. G. y Slaney, R. B., (2002). Clusters of Perfectionists: Two Studies of Emotional Adjustment and Academic Achievement. Measurement and Evaluation in Counseling and Development, 35, 35-48. Recuperado de https://goo.gl/n51BGw

Rice, K. G. y Stuart, J. (2010). Differentiating Adaptive and Maladaptive Perfectionism on the MMPI-2 and MIPS Revised. Journal of Personality Assessment, 92(2), 158-167. doi: http://dx.doi.org/10.1080/ 00223890903 510407

Rosenberg, M. (1965). Society and The Adolescent Self-image. Princeton, NJ: Princeton University Press.

Scotti, D., Sansalone, P. y Borda, T. (2011). Autoestima, miedo a la evaluación negativa y perfeccionismo: un estudio comparativo en población clínica y en población general. Comunicación presentada en el III Congreso Internacional de Investigación y Practica Profesional en Psicología, xvin Jornadas de investigación, séptimo encuentro de investigadores en Psicología del Mercosur, Buenos Aires. Recuperado de https://goo.gl/9uBsZv

Slaney, R. B., Rice, K. G., Mobley, M., Trippi, J. y Ashby, J. S. (2001). TThe Revised Almost Perfect Scale. Measurement and Evaluation in Counseling and Development, 34, 130-145. Recuperado de https://goo.gl/bhzGPJ

Stoeber, J. y Otto, K. (2006). PPositive Conceptions of Perfectionism: Approaches, Evidence, Challenge. Personality and Social Psychology Review, 10, 295-319. doi: http://dx.doi.org/10.1207/s15327957pspr1004_2

Suh, H., Gnilka, P. B. y Rice, K. G. (2017). Perfectionism and Well-being: A Positive Psychology Framework. Personality and Individual Differences, 111, 25-30. doi: http://dx.doi.org/10.1016/j.paid.2017.01.041

Terry-Short, L. A., Owens, R. G., Slade, P. D. y Dewey, M. E. (1995). Positive and Negative Perfectionism. Personality and Individual Differences, 18, 663-668. doi: http:// dx.doi.org/10.1016/0191-8869(94)00192-U 
Trujillo, G. A. (2010). Trabajamos la autoestima y autoconcepto. Revista Digital Tranversalidad Educativa, 40, 29-39.

Wang, K. T., Slaney, R. B. y Rice, K. G. (2007). PPerfectionism in Chinese University Students from Taiwan: A Study of Psychological Well-Being and Achievement Motivation. Personality and Individual Differences, 42(7), 1279-1290. doi: http://dx.doi.org/10.1016/j. paid.2006.10.006
Wong, D. F. K., Chan, K. S. y Lau, Y. (2010-2011). The Role of Perfectionism and Dysfunctional Attitudes in Depression and Quality of Life of a Community Sample of Chinese Adults in Hong Kong. International Journal of Mental Health, 39(4), 40-58. doi: http://dx.doi. org/10.2753/IMH0020-7411390403 